\title{
Analyse de l'activité à bord de dragues aspiratrices : une méthodologie exploratoire combinant données psychologiques et physiologiques
}

Activity analysis aboard dredgers: an exploratory methodology combining psychological and physiological data

Bastien Sennegon, Benoit Grison, Philippe Ravier et Olivier Buttelli

\section{OpenEdition}

\section{Journals}

Édition électronique

URL : http://journals.openedition.org/activites/357

DOI : 10.4000/activites.357

ISSN : 1765-2723

Éditeur

ARPACT - Association Recherches et Pratiques sur les ACTivités

\section{Référence électronique}

Bastien Sennegon, Benoit Grison, Philippe Ravier et Olivier Buttelli, « Analyse de l'activité à bord de dragues aspiratrices : une méthodologie exploratoire combinant données psychologiques et physiologiques », Activités [En ligne], 9-2 I Octobre 2012, mis en ligne le 15 octobre 2012, consulté le 19 avril 2019. URL : http://journals.openedition.org/activites/357 ; DOI : 10.4000/activites.357

\section{cc)}

Activités est mis à disposition selon les termes de la licence Creative Commons Attribution - Pas d'Utilisation Commerciale - Pas de Modification 4.0 International. 


\title{
Analyse de l'activité à bord de dragues aspiratrices : une méthodologie exploratoire combinant données psychologiques et physiologiques
}

\author{
Bastien Sennegon
}

Pôle Nekoé, 16 rue Léonard de Vinci, 45074 Orléans cedex 2, bastiensennegon@yahoo.fr

\section{Benoit Grison}

Faculté du sport et de l'éducation physique, rue de Vendôme - BP 6237 - 45062 Orléans cedex 2,

benoit.grison@univ-orleans.fr

\section{Philippe Ravier}

Laboratoire Pluridisciplinaire en Ingenierie des Systèmes et Mécanique Energétique (PRISME) EA 4229, Polytech Orléans, 12 rue de Blois - BP 6744 - 45067 Orléans cedex 2, philippe.ravier@univ-orleans.fr

\section{Olivier Buttelli}

Laboratoire PRISME EA 4229, Université d’Orléans, olivier.buttelin@univ-orleans.fr

\begin{abstract}
Activity analysis aboard dredgers: an exploratory methodology combining psychological and physiological data. The purpose of this study is to explore a methodology combining two types of data, psychological and physiological, through an ergonomic analysis of the activity. Two field studies were conducted: the stationary suction dredger situation, and the moving suction dredger situation, where we focused on the bridge assistant, responsible for the dredging activity.
\end{abstract}

Two orders of data are considered: psychological and physiological. From a psychological point of view, our methodology cross-referenced ethnographic observations with nondirective interviews. At the physiological level, heart-rate measurements (R-R interval) were continuously recorded, throughout the activity.

Our results proceed from the confrontation of these "objective" and "subjective" data sources. The workload at this work station appears to be considerable, with many short actions. It would seem necessary to maintain a high level of vigilance, due to the various parameters to be taken into account, and to the multiple adjustments which must be made during the task time. The activity can become more complex, depending on the nature of the seabeds: many variations can take place, and there may be occurrences of stress when dredging chaotic seabeds.

This "integrated" approach to human activity should lead to improved safety for the sailors.

\section{KEYWORDS}

analysis of activity, psychophysiology, heart rate variability, case study, safety 


\section{1.- Introduction}

Cette étude ergonomique n'a pas été suscitée par une demande explicite du personnel navigant des dragues aspiratrices, mais fut suggérée par le service de santé des gens de mer. De fait, le milieu maritime est défini comme un secteur professionnel à haut risque, l'activité de travail s'y déroulant en situation d'isolement et au sein d'un environnement naturel difficilement maîtrisable (Le Roy \& Breuille, 1997). Minguy et Chauvin (1998) soulignent également le contexte de travail particulièrement ardu, dans lequel les gens de mer vont évoluer de jour comme de nuit. Dans ces conditions, les enjeux de sécurité paraissent étroitement liés à l'environnement de travail et à l'activité des marins. La Direction Générale de la Mer et des Transports a d'ailleurs répertorié 3400 accidents du travail maritime sur l'année 2007 pour une population de 30343 marins. Cette problématique de la sécurité en milieu maritime est sous-tendue par des liens multiples entre le contexte, l'activité, et les facteurs de risque.

Les difficultés rencontrées par les gens de mer sont fréquemment rapportées par les experts dans leurs analyses de l'accidentologie (Bureau Enquêtes Accidents - mer, 2007). Malgré tout, de telles méthodes d'étude, qui prônent une reconstitution a posteriori des accidents, semblent insuffisantes pour des fins de prévention et de maintien de la sécurité. Journé (2006) dans le cadre de l'industrie nucléaire, émet des réserves quant à la validité des enquêtes post-accidents. Il préconise alors un travail de recherche en amont des situations accidentelles, par l'analyse « du fonctionnement quotidien d'organisations qui gèrent des situations à risques » (Journé, 2006, p. 100).

$\mathrm{Au}$ regard de ces éléments de contexte, l'activité quotidienne des marins nous a paru être un terrain fertile pour explorer une méthodologie d'analyse au croisement de recueils de données psychologiques et physiologiques. En effet, une étude de cas dans cet environnement de travail à haut risque pourrait laisser entrevoir, à l'interface des deux ordres de données, des pistes de régulations en termes de sécurité.

\section{2.- Cadre théorique}

Notre recherche s'inscrit dans la tradition de recherche de l'analyse de l'activité telle qu'elle s'est développée au sein de l'ergonomie francophone de ces dernières décennies. Dans cette perspective, l'activité (caractéristiques de l'opérateur et de la tâche) occupe un statut central dans l'analyse ergonomique du travail (Leplat, 2000). Néanmoins, l'analyse ergonomique de l'activité ne se centre pas strictement sur l'acteur, mais veille à intégrer le contexte «écologique » au sein duquel la tâche est effectuée.

L'analyse de l'activité est devenue un objet de recherche investi par les courants théoriques récents de l'«action située » et de l'« anthropologie cognitive» (Grison, 2004). Sève, Saury, Theureau et Durand (2002), soulignent à ce propos que l'«action située » présente l'avantage d'analyser l'activité humaine en respectant l'environnement de la situation. Cette approche postule que les processus cognitifs ne se développent que dans un contexte situationnel donné. Ainsi, pour Varela (1996), les processus cognitifs ne résident plus «dans la tête » des sujets, mais entre l'acteur et la situation, lesquels s'influencent mutuellement. À la suite de celui-ci, Theureau $(2004,2006)$ reprend la notion de «couplage structurel », résultat de l'interaction entre l'individu et son environnement. De par ce couplage, les processus cognitifs mettent à contribution les multiples ressources de l'environnement, dans leurs dimensions physique ou matérielle, mais aussi sociale et culturelle (Salembier, Theureau, Zouinar, \& Vermersch, 2001).

Nous présupposerons donc ici que l'environnement de travail constitue un lieu privilégié d'étude de l'activité humaine. Nous parlerons alors de situation écologique, l'action étant organisée en fonction de cet environnement (Béguin \& Clot, 2004). Il apparaît alors impératif d'appréhender l'activité dans son contexte naturel de réalisation (Jeffroy, 
Theureau, \& Haradji, 2006), ce qui permet également de respecter l'inscription temporelle du déroulement de l'activité (Leplat, 2008).

S'il nous semble incontournable de décrire et analyser l'activité des marins à bord de leurs navires, cette analyse in situ implique une prise en compte et un ajustement nécessaires visà-vis des contraintes multiples du milieu maritime (Maline \& Dorval, 1991a; Minguy \& Chauvin, 1998). De fait, cet environnement complexe d'accès peu aisé suppose une adaptation méthodologique de la part du chercheur, quitte à élaborer des combinaisons méthodologiques nouvelles pour le recueil des données. Les méthodes d'analyse ergonomiques doivent rester opérantes dans ce milieu d'investigation particulier, d'où un aménagement de méthode indispensable, quitte à abandonner pour cela les apparences de la «science dure » (Theureau \& Jourdan, 2002).

Sous le vocable «étude de cas», Leplat $(2002,2008)$ s'est référé à des recherches ergonomiques se plaçant dans une optique «compréhensive». À la différence des expérimentations, l'étude de cas fournit une analyse approfondie de l'activité en tant que telle. Ainsi, de telles études de terrain privilégient plutôt la validité interne et la validité individuelle au détriment d'une portée de généralisation. Notre démarche se limitera donc à un nombre restreint de marins étudiés. Néanmoins, l'analyse de cas n'exclut pas nécessairement toute forme de validité externe, les résultats obtenus pouvant par exemple suggérer une convergence inter-individuelle entre les sujets de cas distincts.

\section{Vers une approche intégrée de l'analyse de l'activité humaine}

Dans cette étude exploratoire, il s'agira ainsi de construire une méthodologie adéquate, respectant les contraintes physiques, culturelles et techniques inhérentes à l'environnement maritime.

L'observation directe ou encore «ethnographique» est un outil méthodologique régulièrement utilisé dans le cadre de l'analyse de l'activité (Leplat, 2000). Cette méthode d'observation est d'autant plus à privilégier en milieu maritime où les embarquements se caractérisent par leur longue durée (Hazelhurst, 1999), et l'intrication des vies professionnelle et personnelle (Maline \& Dorval, 1991a).

Pour autant, l'observation directe ne suffit pas pour documenter le recueil des données subjectives sur l'activité des marins, ne donnant pas accès aux perceptions du sujet au cours de l'action (Theureau, 2004).

Il est alors important d'y adjoindre des données recueillies sous forme de verbalisations. Ces entretiens individuels autorisent la prise de conscience d'un individu relativement à ses tâches quotidiennes (Vermersch, 2000). Des interviews seront effectuées a posteriori de l'activité, cette démarche rétrospective permettant de corroborer l'observation antérieure par les verbalisations. Ainsi, des données issues de sources variées pourront s'avérer de nature complémentaire (Vermersch, 2006).

Enfin, nous tenterons d'associer un autre type de recueil aux deux catégories de données précédentes, décrivant l'activité d'un point de vue physiologique. Dans le même esprit, Theureau (2006) conseille de croiser plusieurs méthodes, afin de recouper des données issues de sources distinctes et variées. Leplat souligne quant à lui que l' «étude de cas, comme celle de l'activité, échappe à une vue purement disciplinaire. Le cas est au confluent de déterminants multiples qui ne relèvent pas tous du même champ» (Leplat, 2002, p. 3). Deux sources de données seront alors privilégiées dans cette perspective exploratoire, centrée sur un faible effectif d'individus :

— « subjectives », faisant référence à l'observation ainsi qu'aux verbalisations ;

— «objectives », en relation avec l'activité physiologique de l'organisme.

Nous avons choisi la fréquence cardiaque comme indicateur physiologique. Elle offre 
l'avantage de donner lieu à une méthode de mesure non-invasive (électrodes de surface) et peu encombrante. L'enregistrement de ces données de fréquence cardiaque peut donc se dérouler au cours de l'activité quotidienne des sujets. Il était alors question de recouper ces données de fréquence cardiaque, selon une activité précise et un moment déterminé, ce qui est cohérent avec l'approche « compréhensive » et « contextuelle» adoptée.

Les procédures d'analyse de la fréquence cardiaque sont variées. En ergonomie, l'on peut citer le calcul du coût cardiaque, qui est défini par «la somme des pulsations au-dessus $d u$ niveau de repos, rapportée à la période de travail considérée et exprimée en battements par minute» (Monod \& Kapitaniak, 2003). Celui-ci présente l'intérêt d'être opérant in situ (Chassagnieux, Garrigou, Lortie, Teiger, Carbaledda, Pargade et al., 2012) et permet une évaluation de l'astreinte physique. Dans le cas d'une activité dynamique, le coût cardiaque peut être relié au coût énergétique.

Le calcul du coût cardiaque nécessite un moyennage des valeurs de fréquence cardiaque, ou encore une valeur pic peut être prise comme valeur limite. Dans le cadre de la présente étude, il était nécessaire de réaliser un suivi chronologique précis de la réponse cardiaque, au travers d'un indicateur représentatif des différents registres de l'activité du marin, ceci afin d'articuler données physiologiques et psychologiques.

Aussi avons-nous envisagé, dans une démarche de type exploratoire centrée sur la dynamique de l'activité, d'avoir recours à l'analyse de la variabilité de la fréquence cardiaque en dépit de sa complexité.

La variabilité de la fréquence cardiaque est principalement sous la dépendance de la régulation du système nerveux autonome (Rajendra Acharya, Paul Joseph, Kannathal, Choo Min Lim, \& Jasjit Suri, 2006). Cette régulation est réalisée par les deux subdivisions fonctionnelles antagonistes du système nerveux autonome. La branche orthosympathique (ou sympathique) et la branche parasympathique, qui accélèrent et ralentissent respectivement le rythme cardiaque (ibid.). Ces deux branches opèrent simultanément, chacune étant plus ou moins prédominante suivant les conditions environnementales et l'état de fonctionnement de l'organisme. La branche parasympathique est plus prédominante lors de situations de repos, tandis que celle orthosympathique est plus prédominante lors d'activités physiques ou de situations d'urgence (Borresen \& Lambert, 2007; Martinmäki, Häkkinen, Mikkola, \& Rusko, 2008 ; Perini \& Veicstenas, 2003). C'est donc un équilibre entre l'activité des deux branches qui définit la régulation opérée par le système nerveux autonome; au niveau cardiaque cet équilibre est défini par la balance sympatho-vagale.

Différents indicateurs peuvent être calculés afin de caractériser la variabilité de la fréquence cardiaque. Il est souvent fait usage des quantifications énergétiques calculées sur la représentation de la densité spectrale de cette activité (Task Force of European Society of Cardiology, 1996). Sur cette représentation, deux bandes de fréquences distinctes dénommées basse fréquence (LF) et haute fréquence (HF) ont été définies comme représentatives de l'activité de la balance sympatho-vagale (Rajendra Acharya et al., 2006). Dans la bande HF s'exprime principalement l'activité de la branche parasympathique ; dans celle LF s'exprime l'activité de la branche orthosympathique. Cependant, la bande LF est également sensible à la branche parasympathique. C'est pourquoi le rapport des quantités énergétiques de LF sur celles de HF est souvent réalisé (LF/HF), afin de définir un indicateur sensible principalement à l'activité de la branche orthosympathique (Task Force of European Society of Cardiology, 1996).

De nombreux facteurs peuvent avoir une incidence sur la balance sympatho-vagale, et donc modifier les contenus énergétiques des bandes LF et HF. Par exemple, Pierpont, Stolpman et Gornick (2000) ont relevé lors d'un effort physique maximal une augmentation de LF relative à HF. Le stress est un autre facteur influençant la balance sympatho-vagale. Un stress ponctuel sollicite prioritairement la subdivision sympathique du système nerveux autonome - cf. la revue de littérature de Rajendra Acharya et al. (2006). Il est notamment 
observé un accroissement du ratio $\mathrm{LF} / \mathrm{HF}$, conséquence d'une baisse de l'énergie dans la bande HF (Hjortskov, Rissen, Blangsted, Fallentin, Lundberg, \& Sogaard, 2004).

La variabilité de la fréquence cardiaque est donc dépendante de nombreux facteurs. Les travaux utilisant cette variabilité nécessitent de prendre en compte et/ou de contrôler ces facteurs, quand l'objet de l'étude porte sur l'un de ceux-ci.

L'analyse de la variabilité dans le domaine fréquentiel nécessite un calcul de transformée. Les procédures (transformées) pour passer du domaine temporel au domaine fréquentiel sont multiples. Le choix de ces méthodes de calcul devrait se faire en fonction de la nature du signal et des gammes fréquentielles analysés (Rajendra Acharya et al., 2006 ; Task Force of European Society of Cardiology, 1996).

Dans le cas de la variabilité de la fréquence cardiaque, deux gammes fréquentielles sont analysées ; la gamme HF $[0,15 \mathrm{~Hz}-0,3 \mathrm{~Hz}]$ et la gamme LF $[0,04 \mathrm{~Hz}-0,15 \mathrm{~Hz}]$.

Lorsque l'information temporelle doit être conservée conjointement avec l'information fréquentielle, il est intéressant de faire appel à des représentations temps/fréquence conjointes. Il existe de nombreuses méthodes pour réaliser de tels calculs, mais c'est la nature du signal analysé qui devrait définir le calcul le plus approprié pour effectuer ce type de transformée.

Les signaux bioélectriques sont considérés comme un signal très peu stationnaire. Leurs caractéristiques statistiques sont variables dans le temps et dépendent des instants d'observation (Guzzeti, Signorini, Cogliati, Mezzeti, Porta, Cerutti et al., 1996). Dans ce cas, il est préférable d'utiliser une méthode qui tienne compte de cette propriété, afin de réduire les erreurs liées au calcul de transformée. L'analyse «en ondelettes» est une de ces méthodes (Samar, Bopardikar, Rao, \& Swartz, 1999). Le terme ondelette fait référence à des petites ondes au travers desquelles le signal analysé est décomposé en différents coefficients, qui permettent de recomposer un signal dans l'espace temps/fréquence (Grossmann \& Morlet, 1984).

Cette méthode de calcul sera donc utilisée au cours du suivi de l'activité cardiaque, dans l'intention d'observer les différentes réponses du système nerveux autonome, ce en fonction de la survenue des événements pendant toute la durée d'une tâche donnée.

\section{La mise en place d'« interfaces opératoires »}

Enfin, nous allons tenter de circonscrire des interfaces opératoires entre les différents ordres de données de cette approche intégrée de l'analyse de l'activité. Initialement, ces données, issues de champs scientifiques différents, ne sont pas produites pour être confrontées entre elles. Outre une analyse « rapprochée » de l'activité, nous chercherons à prévenir d'éventuels décalages entre la réalité et la perception qu'en a le marin. Néanmoins, nous ne sousestimons pas la difficulté de cet exercice, du fait du caractère hétérogène des données.

Un tel positionnement théorique n'autorise pas un approfondissement des niveaux d'analyse à degré égal, par rapport aux chercheurs se consacrant à un niveau unique. Mais nous faisons ici le pari de la fécondité d'une construction méthodologique étayée sur plusieurs niveaux d'analyse. Ce dispositif exploratoire pourrait ainsi faire émerger, à l'interface des niveaux, des conclusions scientifiques en partie inaccessibles à partir d'une seule perspective de méthode.

Pour ce faire, dans le cadre de cette approche «intégrée », nous allons tenter de circonscrire des interfaces opératoires entre les différents types de données. Cette «triangulation» des données implique d'articuler nos différents niveaux d'analyse, et non de les juxtaposer (Leplat, 2000). Il devient alors nécessaire d'instaurer un «dénominateur commun» méthodologique, afin de mettre les données psychologiques et physiologiques, perçues et objectivées, en interrelation. 
La disparité initiale des niveaux d'analyse, manifeste, viserait à se transformer ici en « richesse » ou complémentarité d'analyse. Dans l'intention d'appréhender une situation de la manière la plus globale et précise qui soit, à travers une diversité des outils méthodologiques employés.

\section{3.- Méthodologie}

\section{Le terrain d'étude}

Le Port Autonome de Nantes Saint Nazaire possède deux dragues aspiratrices. Leur rôle est de retirer la vase, le sable, ou encore les graviers qui s'amoncellent sur les fonds marins, et perturbent le bon fonctionnement des installations portuaires.

- La première drague est dite aspiratrice stationnaire, car elle ne dispose pas de moteur de propulsion et officie exclusivement dans l'estuaire de la Loire. Néanmoins elle peut se déplacer sur de courtes distances (quelques centaines de mètres) :

— latéralement avec des treuils enroulant des câbles (reliés à des ancres ou à la berge) ;

— en avançant ou reculant, s'appuyant alternativement sur deux pieux inclinables de $30 \mathrm{~m}$ de long situés à l'arrière du navire.

Le système d'aspiration de la drague est composé d'une élinde (bras articulé) située à l'avant de l'embarcation et descendant au fond de l'eau, pour aspirer la vase et broyer les roches ou métaux avec un désagrégateur ( «cutter»). La drague rejette simultanément la vase aspirée au milieu de l'estuaire de la Loire, via une conduite souple.

Le mode de fonctionnement de la drague est ininterrompu, son personnel travaillant en «bordées » (équipes) selon des rotations quotidiennes $(7 \mathrm{~h}$ et $19 \mathrm{~h}$ ). L'observateur a donc embarqué deux semaines sur la drague stationnaire : une pour suivre le travail de jour, l'autre pour suivre le travail de nuit. Nous nous sommes focalisés sur l'activité de l'assistant de pont qui était personnellement en charge du dragage (désigné ici sous le pseudonyme de Rémy). Son poste de travail se situait au centre de la passerelle, face à l'élinde qu'il commandait. Il disposait aussi des manettes des pieux et des treuils pour faire évoluer la drague sur le chantier.

- La seconde drague est dite aspiratrice en marche, car elle se déplace de manière autonome. Elle officie dans les ports ou les estuaires, en extrayant du sable, des roches ou de la vase qu'elle stocke dans ses cuves. Elle va ensuite déverser ce contenu dans des fosses sous-marines. La passerelle (poste de pilotage) est implantée à l'avant, tandis que les cuves à ciel ouvert sont centrales. L'élinde est déportée sur l'arrière droit.

De la même manière que celle de type stationnaire, cette drague fonctionne sans interruption. Cependant il n'y a qu'une bordée (travail jour et nuit sous forme de quarts de veille), la relève s'effectuant toutes les semaines. Sur l'aspiratrice en marche, l'observateur est resté une semaine, la zone de travail étant l'estuaire de la Seine, puis l'avant-port de Boulogne sur Mer. L'activité de l'assistant de pont (désigné ici sous le pseudonyme de Jimmy) y fut également étudiée. Son pupitre était dos à la marche, afin d'être face à l'élinde qu'il dirigeait par ses multiples commandes. Ses horaires de travail étaient de $0 \mathrm{~h}$ à $6 \mathrm{~h}$ et de $13 \mathrm{~h}$ à $19 \mathrm{~h}$ pour la semaine observée.

\section{Le recueil des données}

\section{- L'observation directe}

L'observation ethnographique directe a consisté à participer «in vivo » à l'activité professionnelle des marins, le chercheur s'immergeant dans le terrain d'étude (Becker, 1958 ; Gold, 1958). Cette approche, à travers l'empan temporel long adopté, permet une description fine et rigoureuse des comportements (Laperrière, 1992). Initialement cet outil 
est usité en ethnologie, mais ici, ce type d'observation sera employé à des fins ergonomiques (Geslin, 2002). Il vise à recueillir des données empiriques sur l'activité des marins dans leur «monde réel » (Hazelhurst, 1999), au sein de l'univers intersubjectif partagé qui est le leur (Schütz, 2009).

La situation d'observation participante est portée à son paroxysme à bord d'un bateau : forte proximité, longue durée d'embarquement et impossibilité de sortir du terrain (Duval, 1998 ; Maline \& Dorval, 1991a). Dans ce cas, la «familiarisation» avec celui-ci (relations humaines et matérielles) constitue a fortiori une étape ethnographique cruciale.

Simultanément à l'activité des marins, l'observateur a pris des notes sur un carnet ethnographique. Ce carnet regroupait les notes provenant de l'observation, de discussions, ainsi que des analyses esquissées dans le but de comprendre l'activité.

Concernant son déroulement chronologique, l'observation directe a été déclinée en plusieurs étapes (Bachelor \& Joshi, 1986 ; Becker, 1958) :

— description systématique des phénomènes et informations sur l'organisation ;

— évaluation de la récurrence des phénomènes observés et de leur fréquence d'apparition ;

— construction des catégories d'analyse pour rendre compte de l'organisation générale de la situation (cette phase appartient déjà pour partie au traitement des données).

\section{- Les entretiens}

Les entretiens ont pour finalité de faire émerger des «données expérientielles brutes ». Ces témoignages verbaux vont constituer des descriptions détaillées d'événements et processus vécus (Bachelor \& Joshi, 1986). Ainsi, les entretiens ne furent pas directifs, mais compréhensifs (non-directifs). La discussion débutait par une question d'ordre général nonsuggestive, alors que par la suite, les questions portaient exclusivement sur des demandes d'apports d'informations et de précisions. Tous les entretiens furent intégralement enregistrés à l'aide d'un dictaphone.

Deux entretiens d'une heure maximum ont été réalisés par sujet. Ils eurent lieu à bord du navire, à la suite de trois jours minimum d'observation. Ces entretiens n'ont donc en aucun cas été improvisés, mais ressemblaient plutôt à des éclaircissements et confirmations éventuelles relatifs à l'observation antérieure. En ce sens, le fait d'effectuer les entretiens à la suite des observations facilite le déroulement des premiers. Cet ancrage dans la réalité des situations observées autorise une contextualisation systématique lors des recueils de données de verbalisations.

Pour les seconds entretiens, nous avons dû opérer une régulation, au vu du caractère «peu disert » de la culture des gens de mer. Nous avons alors utilisé les enregistrements vidéo de l'activité des marins (initialement réalisés pour faciliter l'interprétation des enregistrements de la fréquence cardiaque évoqués plus loin). Ainsi, l'observateur visionnait la bande vidéo préalablement au deuxième entretien, et sélectionnait une séquence de cinq minutes caractéristique de l'activité du sujet. Au début de l'entretien, il diffusait cette séquence choisie au sujet et lui demandait de décrire son activité. Toutefois, nous n'avons eu recours en aucun cas à des autoconfrontations (Theureau, 2004, 2006 ; Theureau \& Jeffroy, 1994), l'objectif visé étant essentiellement de faciliter la verbalisation des marins sur la base d'un exemple concret. L'entretien débutait par les commentaires du marin sur son activité (durant moins de 15 minutes), et se poursuivait comme indiqué précédemment par un entretien compréhensif.

La dernière phase dans la production des données verbales fut leur retranscription intégrale. L'observateur a réécouté les enregistrements, puis reporté systématiquement toutes les verbalisations par écrit au moyen d'un logiciel de traitement de texte.

\section{- La variabilité de la fréquence cardiaque}

Les appareils de mesure utilisés étaient des «cardiofréquencemètres POLAR S810i®», 
l'étude de Cassirame, Tordi, Mourot, Rakobowchuk et Regnard (2007) ayant montré la fiabilité de ce dispositif.

Le cardiofréquencemètre est composé de :

- deux électrodes de mesure intégrées sur une ceinture portée au niveau du thorax ;

— une montre/récepteur qui enregistre les données captées par les électrodes.

Suite à l'enregistrement, le transfert des données de la montre vers l'ordinateur s'effectue via un port infrarouge. Les données étaient enregistrées puis stockées au moyen du logiciel «Polar Précision Performance SW® ».

Les enregistrements de la fréquence cardiaque étaient réalisés au cours de l'activité des marins, la mesure étant prise sur une durée d'une heure, à différentes périodes de l'embarquement. Nous avons effectué quatre enregistrements avec Rémy (drague stationnaire) : le $01 / 10$ à 9 h 57, le $03 / 10$ à 15 h 55, le 14/10 à 1 h 59, et le $17 / 10$ à 1 h 6 . Ensuite, nous avons réalisé deux enregistrements avec Jimmy, le 27/10 à 15 h 57 et le 30/10 à 15 h 15 .

Simultanément à l'enregistrement de la fréquence cardiaque, l'observateur relevait les différentes actions du sujet observé dans son carnet ethnographique (horaire de début et durée de l'action). Un enregistrement vidéo de l'activité du sujet était également réalisé. Les notes ethnographiques ainsi que les enregistrements vidéo s'avèrent fondamentaux pour l'interprétation des données de la fréquence cardiaque (Maline \& Dorval, 1991 b).

\section{Le traitement des données}

\section{La variabilité de la fréquence cardiaque}

Les données furent exportées du logiciel « Polar ${ }^{\circledR} »$ au format texte, vers l'environnement de développement du logiciel Matlab 6.5®. Dans cet environnement, différents scripts développés avec l'équipe Signal du laboratoire PRISME de l'Université d'Orléans, ont permis l'analyse de la variabilité de la fréquence cardiaque.

Les signaux contenant les intervalles R-R ont été traités à partir d'une transformée en ondelette continue de Morlet (Rajendra Acharya et al., 2006). Les quantités d'énergie correspondant aux deux bandes HF et LF furent exprimées en unité arbitraire, tout au long de l'enregistrement (échelle temporelle).

Les résultats ont été présentés sous forme de graphique temps/fréquence :

— l'échelle de temps en abscisse (de 0 à 60 minutes) ;

— la fréquence en ordonnée ( $\mathrm{LF}[0,04 \mathrm{~Hz}-0,15 \mathrm{~Hz}]$ et $\mathrm{HF}[0,15 \mathrm{~Hz}-0,3 \mathrm{~Hz}])$;

- les quantités d'énergie ont été représentées par une gamme de couleurs (la quantité augmentant du bleu au rouge).

L'analyse de l'activité sera alors interprétée à travers les régulations du système nerveux autonome. Les enregistrements vidéo et les données ethnographiques aideront à relier les variations d'énergie du graphique avec le descriptif d'activité du marin (contextualisation).

\section{L'observation directe}

Comme nous l'avons souligné précédemment, les phases de recueil et de traitement des données d'observation tendent à s'inscrire dans un continuum (Becker, 1958). Effectivement, lors de la phase de construction des données, l'observateur relisait au fur et à mesure les données recueillies. Ceci avait pour effet de l'amener à approfondir les investigations sur le terrain, afin de vérifier et préciser des ébauches d'analyse.

Le traitement des données du carnet ethnographique s'est réalisé en deux phases distinctes. $\mathrm{Au}$ préalable, il était nécessaire d'intérioriser et de mémoriser toutes ces données par l'intermédiaire de multiples lectures du carnet. Cette première phase de traitement a consisté en un découpage du contenu en différentes catégories de signification (Strauss \& Corbin, 
1990). Puis venait la deuxième phase de traitement, consistant en une analyse plus fine. Il s'agissait de créer des sous-catégories en fonction de la richesse quantitative et qualitative des données. À ce stade du traitement furent dégagées les similitudes et récurrences interindividuelles, au moyen d'une comparaison entre les catégories de signification relatives aux différents marins. Ceci permettant de décrire les «structures fondamentales des phénomènes étudiés » pour reprendre les termes de Bachelor et Joshi (1986). Ces sous-catégories furent par la suite découpées, réorganisées et renommées (Strauss \& Corbin, 1990).

Le traitement des données vidéo et d'observation associées aux enregistrements de la fréquence cardiaque nous a permis quant à lui d'élaborer des mesures quantitatives de l'activité des marins (relevé des différentes tâches sur 60 minutes).

\section{Les entretiens}

En ce qui concerne les entretiens, les données brutes furent traitées de façon similaire à celles issues de l'observation directe. À savoir une première étape de relecture pour assimiler et découper le corpus recueilli; puis une seconde étape avec analyse plus fine, caractérisée par l'émergence de sous-catégories.

\section{L'articulation des différents niveaux d'analyse}

L'ultime phase de traitement des données avait pour objectif de synthétiser les informations provenant des différents niveaux d'analyse. Il s'avérait alors nécessaire de comparer, recouper, et réorganiser les différentes données traitées. D'où l'intérêt heuristique de circonscrire des interfaces opératoires autorisant la réunion de ces données hétérogènes, le but étant de faire dialoguer ces données issues de sources variées.

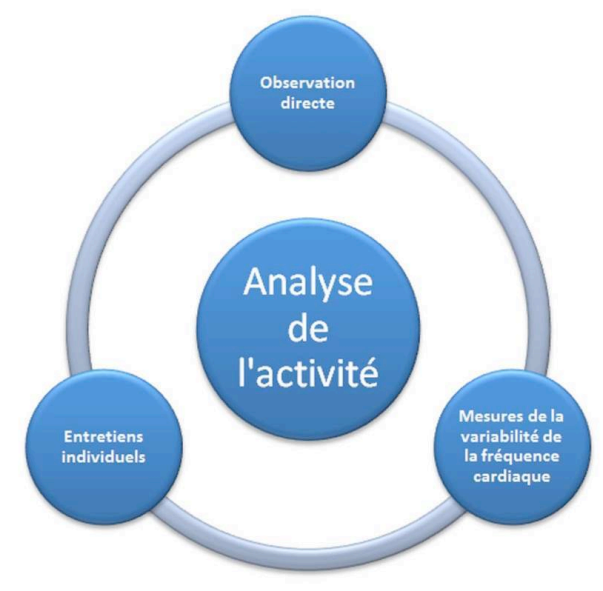

Figure 1: L'activité saisie à travers trois niveaux d'analyse croisés

Figure 1: The activity through three levels of analysis

\section{4.- Résultats}

Les résultats de l'observation ethnographique révèlent de longues durées de travail au poste de dragueur. La durée moyenne du travail quotidien observée sur la drague stationnaire est de 9 h 50 en bordée de jour et 8 h 50 en bordée de nuit. Sur la drague aspiratrice en marche, cette durée moyenne s'élève à 12 h 20, mais est divisée en deux séquences sur 24 h.

L'activité principale du dragueur consiste à piloter le bras articulé (élinde) dont l'extrémité sert à broyer et aspirer le fond marin. Ce bras articulé est commandé au moyen de différents treuils actionnés par des manettes depuis le poste du dragueur. Il s'agira d'épouser le profil $\mathrm{du}$ fond sous-marin avec l'élinde en creusant à la bonne profondeur, tout en recherchant le compromis optimal entre un bon rendement et la non-obstruction du système d'aspiration. Concrètement, plus l'on va draguer rapidement, plus l'on est confronté au risque de boucher 
la conduite d'aspiration. L'activité est dès lors sous-tendue par la nécessité de maintenir un niveau de vigilance élevé.

\section{La vigilance au poste de dragueur}

Simultanément au pilotage de l'élinde, la surveillance de l'assistant de pont s'établit sur une multitude d'instruments (voir Verbalisations 1) pendant toute la durée du quart de travail. Les instruments sont répartis autour du poste de travail : manomètres, compte-tours, alarmes, écrans d'ordinateur (débit, cartographie, représentation de l'élinde...), ainsi que câbles et treuils. Sur la drague stationnaire, l'assistant contrôle de surcroît l'avancée du navire par le biais des pieux.

\section{Verbalisations 1 :}

Rémy : «Là, j'augmente le moteur à 720 tours, donc je regarde mon cadran, pour monter le moteur à 720 , et voilà 720 , là, je suis en train de régler à 700-720, je regarde mes écrans en haut pour voir si le débit est bon, j'amène un peu l'élinde pour pouvoir faire la côte au fond [...] là j'avance, j'arrive en bout de balancé, donc j'avance sur le pieu. [...] Et je vais repartir à draguer, $\mathrm{j}$ 'amène l'élinde un peu, et voilà, et je suis reparti pour la drague, là, je sais pas ce que j'ai fait, le stop là, généralement c'est pas prévu ça, ah, là, là je fais les pieux, là derrière, je suis rendu en bout de queue donc je recommence, je le revire pour le remettre en position de dragage, là je vire. [...] Voilà, et je surveille visuellement les pieux voir s'ils descendent bien, et si ça monte bien, alors là, je dois être en train de le descendre, voilà, là j'incline, je ré-incline le pieux, et je vais le repiquer ensuite, alors je l'incline comme ça. [...] Voilà, alors j'incline, je suis toujours en train de l'incliner là, tac le bruit, là tu vois, et je vire l'autre pieu maintenant, parce que le temps que je fasse un pieu, il faut bien que je mette l'autre pieu au fond, parce que tu as ta drague qui se barre dans le courant. » n.41

La sollicitation de l'assistant de pont se caractérise à travers le nombre de paramètres qu'il doit prendre en considération au cours du dragage (voir Verbalisations 2). Ces multiples focalisations peuvent d'ailleurs entraîner des perceptions de fatigue et de lassitude cognitive.

\section{Verbalisations 2 :}

Rémy : «Les yeux sur les écrans, sur les manos, c'est fatigant ! » n.29

Rémy : «Et regarder les treuils en bas, voir si les câbles, si ça s'enroule bien, et toujours avoir l'œil sur le moteur, les manos du moteur, les alarmes, tu vois tout ce qui, et en plus le dragage, voir si ça touche, donc tu as toujours les yeux dessus !»n.43

Rémy : «Les ordinateurs, tu es toujours en train de regarder, ça te fait plus mal aux yeux la nuit que de jour ! »n.46

Jimmy : «Je regarde surtout le mano d'intensité de la pompe, là, il est très important. » n.57 Jimmy : «Quand on est en manuel plutôt qu'en automatique, souvent on va voir, on se déplace, sur un levier, j' ai deux vitesses. [...] et quand on est en manuel, il faut regarder les longueurs de câble qu'on met dans l'eau. [...] s'il y a pas mal de fond, je vais laisser en automatique, ça joue sur un levier [...] s'il y a un problème, le temps que ça réponde, ce sera trop long. » n.58

\section{Les caractéristiques de la zone à draguer}

La difficulté du dragage réside avant tout dans la composition des fonds marins et la nature des objets susceptibles de s'y trouver (voir Verbalisations 3). En effet, sur des fonds de sable ou de vase, le fonctionnement de la drague va être optimal, avec un bon rendement à travers le rapport surface draguée/durée de dragage. Sur des fonds plus chaotiques, tels que des rochers, l'avancée est bien plus lente. Le dragage s'accompagne de fortes et incessantes vibrations, résultant de la découpe des roches par le cutter.

Verbalisations 3 :

Rémy : «Moi, j'appelle un chantier peinard, un chantier où tu pêches de la vase, t'es content de pêcher de la vase, en plus t'es bien, tu fais du bon boulot, et ça va, ça c'est un chantier peinard ! [...] Quand c'est un chantier comme sur les cailloux, où tu vas bousiller la drague, 
alors tu vois, ça m’énerve, là, ça me gonfle !!!» n.33

Jimmy : «C'est vrai qu'on trouve des cailloux, de la ferraille. » n.59

Rémy : «J'aime bien faire un chantier où je pêche de la vase, ou du sable, mais j'aime pas qu'on me mette sur un chantier, où qu'on pêche de la merde, des cailloux, où c'est sans arrêt bouché, alors là, ça m'énerve. » n.34

De plus, toutes sortes de débris viennent fréquemment obstruer le mécanisme d'aspiration de la drague : des résidus d'appareils de guerre devant le port de Boulogne sur Mer, ou des voitures à proximité de Nantes. Le dragueur réalise des «chasses» pour pallier à ces obstructions. Une «chasse » consiste en l'arrêt de l'aspiration ainsi que la remontée du bec, afin que l'objet obstructif redescende naturellement de la conduite. Lorsque cette remédiation ne s'avère pas suffisante, les matelots doivent déboucher manuellement la conduite, par le biais d'orifices de surveillance, voire en dernier recours, se faufiler dans le tuyau... Un débouchage de ce type va imposer l'arrêt total du dragage (de 15 minutes jusqu'à parfois une heure). Ces obturations, momentanées ou plus conséquentes, surajoutent alors du travail aux marins, notamment pour le dragueur (voir Verbalisations 4).

Verbalisations 4 :

Rémy : «Là, je descends le bec, je suis en train de faire une chasse là, je dois avoir un caillou dans le bec, tu vois, j'ai un caillou dedans, alors là, j'ai stoppé à gauche le moteur, et j'amène mon bec en bas, et le bec descend pour que le caillou puisse tomber, et une fois que ça va être fait. [...] Je relève le bec, et là, je remets en route, je rebalance, je rebalance la machine [...] Voilà, je regarde les manos pour voir si c'est débouché, normalement ça doit être débouché, parce que j'aurais fait une deuxième chasse. » n.41

La tâche du dragueur apparait alors comme répétitive et routinière, car hormis les changements de sites géographiques, l'activité dans sa globalité, organisée autour du pilotage de l'élinde, semble stable et prévisible. Cependant, une analyse affinée autorise une distinction des situations de dragage, à travers l'étude de courtes séquences d'activité. Nous avons ainsi ciblé des séquences d'une heure, représentatives de la tâche du dragueur, c'est-àdire sans apparition d'événements singuliers (fort trafic maritime, contraintes météorologiques, mise à sec d'obus...).

\section{Analyse « micro » de courtes séquences d'activité}

Une forte variation de l'activité du dragueur, en fonction de la nature des fonds sous-marins, peut s'observer à travers l'analyse des représentations temps/fréquence de l'enregistrement de la variabilité de la fréquence cardiaque, notamment chez l'assistant de pont de la drague stationnaire (voir Figure 2). Sur la bande HF, cet enregistrement (Figure 2) présente très peu de variations d'énergie au cours du temps, alors que nous observons quelques accroissements sur la bande LF.

Ces variations d'énergie sont la conséquence d'une augmentation de l'activité de la branche orthosympathique sur celle parasympathique, pour les pics observés dans la bande LF. Par recoupement avec les données ethnographiques, l'on peut affirmer que cet enregistrement correspond à une période de faible activité de l'assistant de pont. Le dragage est arrêté à 11 ', où nous interprétons le pic sur la bande LF comme la dépense physique du passage en position debout de l'assistant de pont quittant son siège. Les deux autres pics qui témoignent de l'augmentation d'énergie sur cette bande sont corrélés avec des discussions : par VHF (42') et orale (55'). 

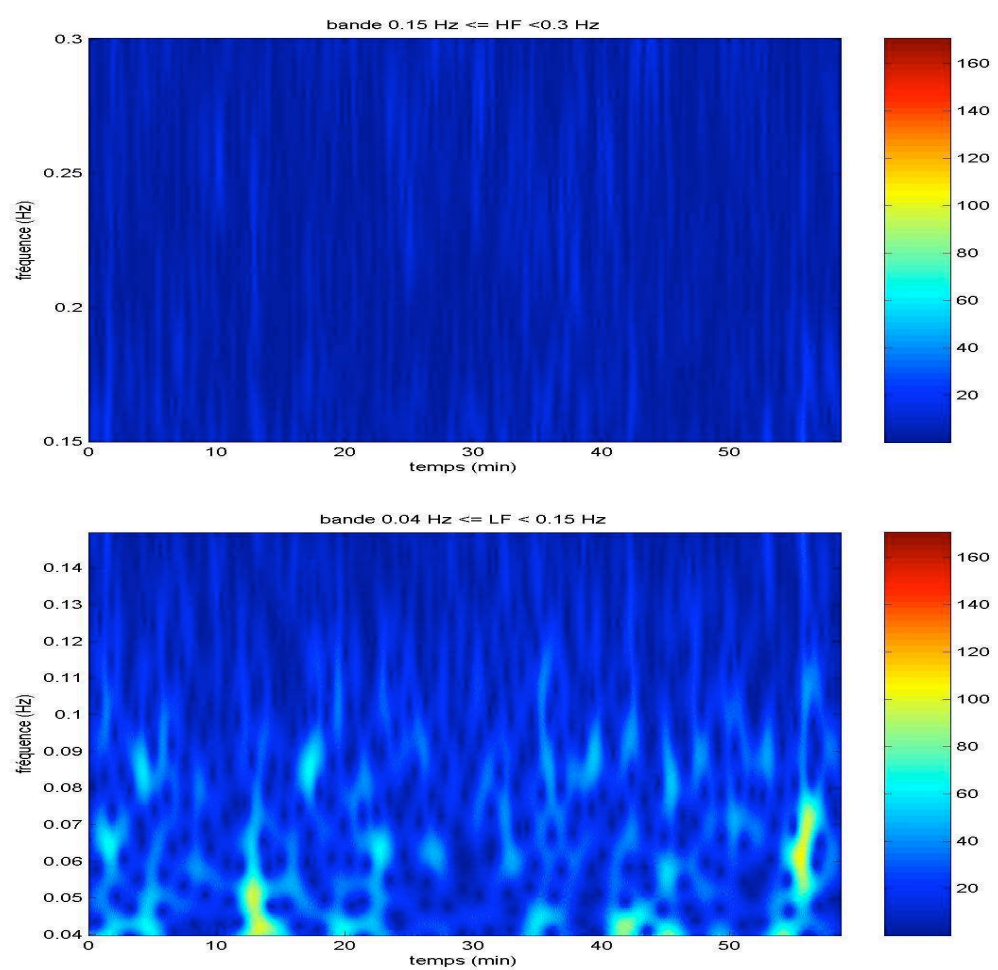

Figure 2 : Représentation temps-fréquence du contenu énergétique d'un enregistrement de la variabilité de la fréquence cardiaque. Enregistrement Rémy

1 , fait le $01 / 10 / 05$, début à $9 \mathrm{~h} 57$. La quantité d'énergie exprimée en unité arbitraire est représentée par les couleurs ; la quantité augmente du bleu au rouge.

Figure 2: Time-frequency graph based on the energy content of a continuous recording of heart rate variability. Rémy 1 recording, dated 01/10/05, starting at 09:57. Energy content is represented by a color scale, quantity increasing from blue to red.

À l'inverse, sur ce nouvel enregistrement de l'assistant de pont de la drague stationnaire (Figure 3), la représentation de la bande LF comporte de nombreuses variations tout au long de l'enregistrement (la bande HF présentant toujours peu de variations en énergie).

La bande LF est alors surchargée. Ces variations d'énergie sont la conséquence d'une augmentation de l'activité de la branche orthosympathique sur celle parasympathique, avec augmentation de ce rapport lors des pics observés dans la bande LF. En recoupant avec les horaires fournis par les relevés ethnographiques, nous notons principalement la réalisation d'une « chasse » de 4' à 8', une longue période de dragage fastidieuse de 15' à 38' (avec une manœuvre de pieux à $30^{\prime}$ ), puis la reprise d'une zone de dragage difficile à $55^{\prime}$ '. Lors de cet enregistrement, le dragueur paraît largement sollicité, à cause d'un fond rocheux, qui exige une vigilance et une réactivité de tous les instants. Ainsi, ce stress ponctuel engendré par l'activité de dragage se répercuterait sur la branche orthosympathique. 

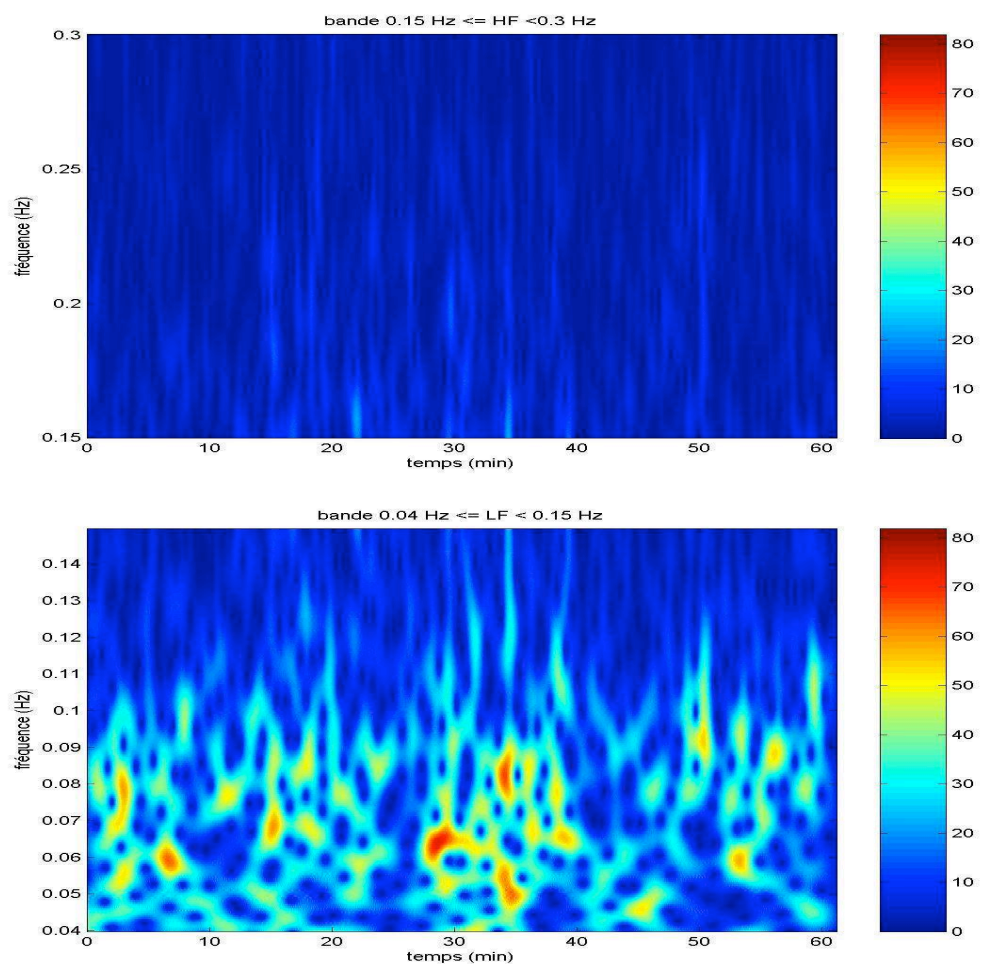

Figure 3 : Représentation temps-fréquence du contenu énergétique d'un enregistrement de la variabilité de la fréquence cardiaque. Enregistrement Rémy

4 , fait le 17/10/05, début à 1 h 6 . La quantité d'énergie exprimée en unité arbitraire est représentée par les couleurs ; la quantité augmente du bleu au rouge.

Figure 3: Time-frequency graph based on the energy content of a continuous recording of heart rate variability. Rémy 4 recording, measured on the 17/10/05, starting at 01:06. Energy content is represented by a color scale, quantity increasing from blue to red.

Cette séquence de dragage difficile pour cause de fond rocheux avait également été repérée lors de l'observation ethnographique, avec 134 actions notées lors de cette séquence du 17/10 (voir tableau 1). Les interventions de l'assistant de pont sur la drague stationnaire sont courtes (quelques secondes), nombreuses (plus de deux actions par minute) et variées (sept régulations différentes, hors communication).

\begin{tabular}{ll}
\hline Description de l'activité & Nombre d'actions \\
\hline Treuils du papillonnage (manettes) & 23 \\
Vitesse des treuils papillonnage (molette) & 31 \\
Treuil élinde (manettes) & 16 \\
Treuil Bec (manettes) & 16 \\
Vitesse de rotation du cutter (manettes) & 16 \\
Manœuvres des pieux (manettes) & 14 \\
Pompe (mise en marche/arrêt) & 15 \\
Communication (VHF, interphone, orale) & 3 \\
\hline Total & 134 \\
\hline
\end{tabular}

Tableau $1:$ La charge de travail de l'assistant de pont sur la drague stationnaire durant une heure d'activité (Observation du 17/10/05, bordée de nuit)

Table 1: Deck assistant's work load on the dredger during one hour of activity (Observations on the 17/10/05, night shif) 
À bord de la drague aspiratrice en marche, nous observons également cette complexification de la tâche de l'assistant de pont, lors de la présence de roches ou de débris sur les fonds marins. À l'aide de l'analyse des représentations temps/fréquence du dragueur, nous pouvons dissocier l'activité sur fond «aisé » (voir Figure 4) de celle sur fond «difficile » (voir Figure 5).
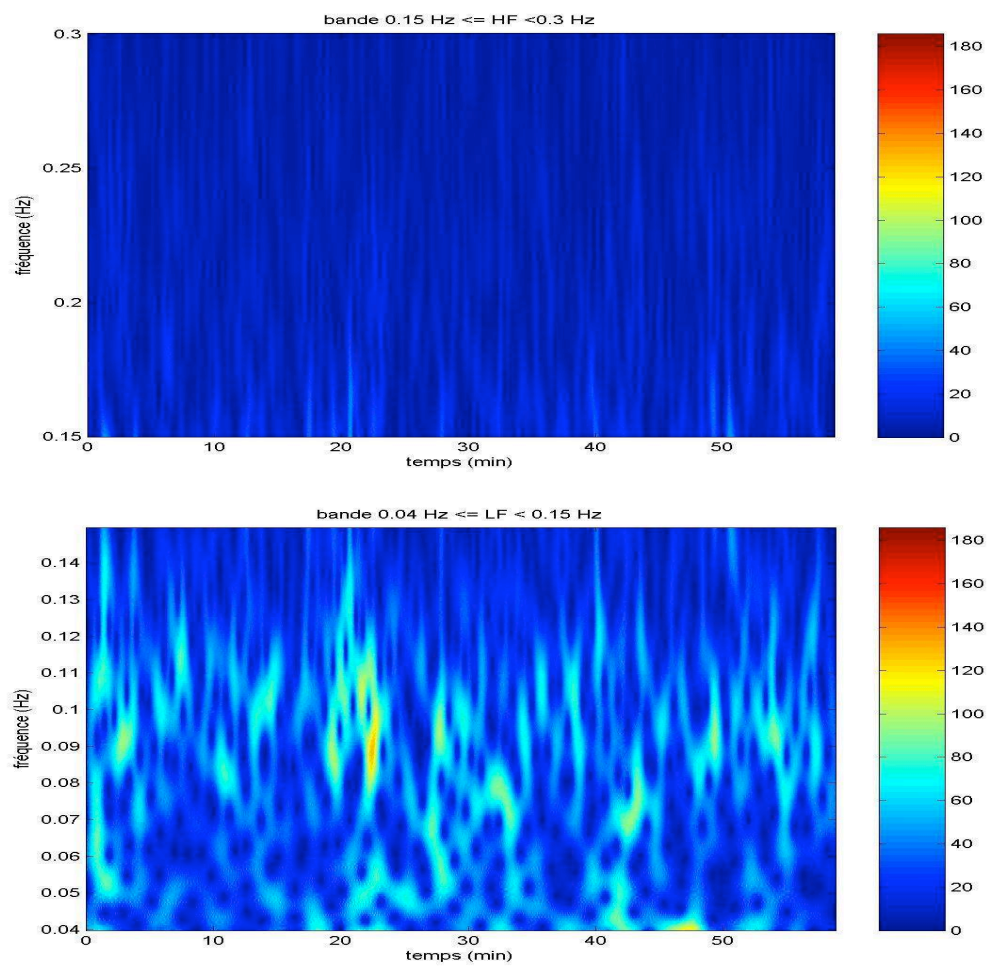

Figure 4 : Représentation temps-fréquence du contenu énergétique d'un enregistrement de la variabilité de la fréquence cardiaque. Enregistrement Jimmy 1 , fait le 27/10/05, début à 15 h 57 . La quantité d'énergie exprimée en unité arbitraire est représentée par les couleurs ; la quantité augmente du bleu au rouge.

Figure 4: Time-frequency graph based on the energy content of a continuous recording of heart rate variability. Jimmy 1 recording, measured on the 27/10/05, starting at 15:57. Energy content is represented by a color scale, quantity increasing from blue to red.

Cette représentation présente peu de variations en énergie au cours du temps sur la bande HF, mais plusieurs accroissements d'énergie sur la bande LF (Figure 4).

Cet enregistrement a été réalisé sur une zone de dragage aisée. En recoupant avec les données ethnographiques, l'on a pu constater que l'assistant n'apportait pas de régulation au mode de dragage automatique (sollicitation des différents treuils), le pic d'énergie en LF à 20' coïncidant avec une discussion.

Cette nouvelle représentation (Figure 5) comporte de très nombreux accroissements énergétiques tout au long de l'enregistrement sur la bande LF (toujours très peu de variations en terme d'énergie en HF). 

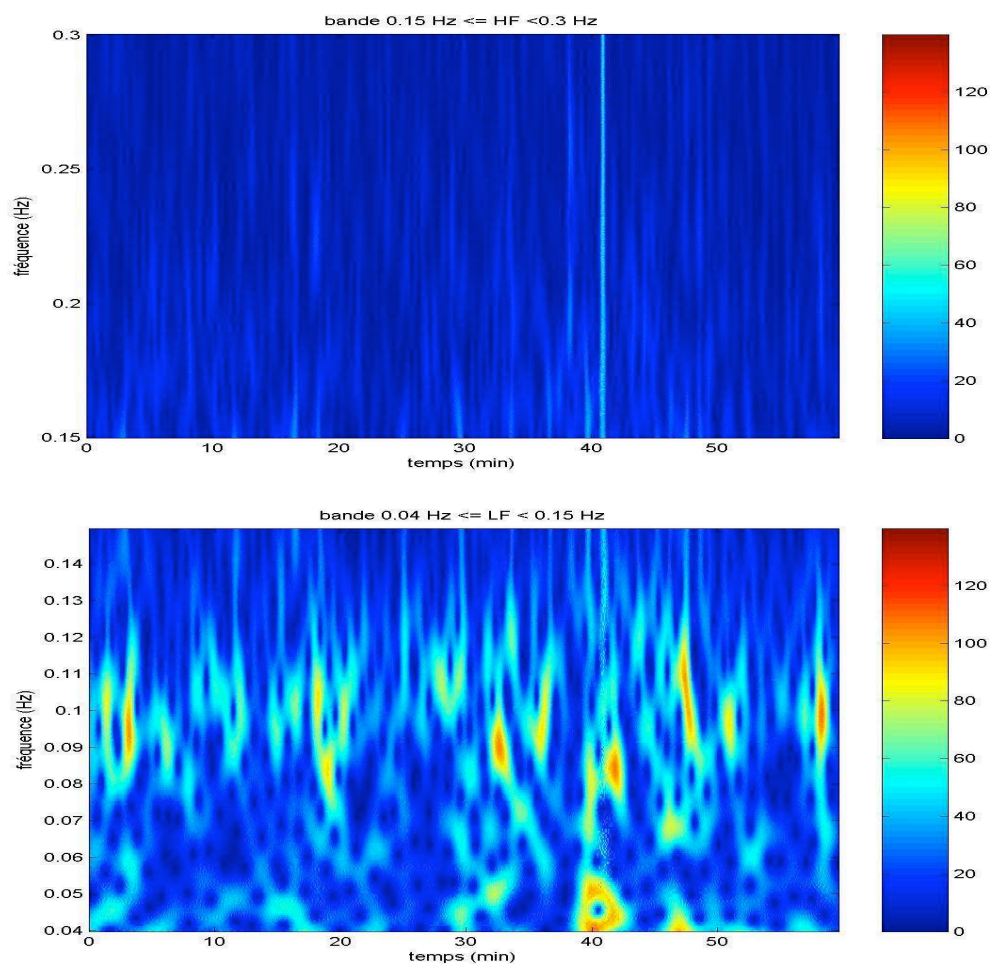

Figure 5 : Représentation temps-fréquence du contenu énergétique d'un enregistrement de la variabilité de la fréquence cardiaque. Enregistrement Jimmy 2, fait le 30/10/05, début à 15 h 15 . La quantité d'énergie exprimée en unité arbitraire est représentée par les couleurs ; la quantité augmente du bleu au rouge.

Figure 5: Time-frequency graph based on the energy content of a continuous recording of heart rate variability. Jimmy 2 recording, measured on the 30/10/05, starting at 15:15. Energy content is represented by a color scale, quantity increasing from blue to red

Il se trouve que cet enregistrement a eu lieu lors d'une activité de dragage difficile, effectuée en mode manuel sur un fond rocheux. Cette intensité du dragage (nécessitant vigilance et réactivité) peut être source de stress ponctuel, et se répercuterait au niveau de la bande LF (sollicitation de la branche orthosympathique). Nous repérons par recoupement avec l'observation ethnographique : la mise à l'eau de l'élinde (4'), une première " chasse » à 20 ', le déclenchement d'une alarme à 32', ainsi qu'une seconde chasse fastidieuse de $40^{\prime}$ à 50' (jusqu'à la remise au fond de l'élinde).

Nous avons recensé 196 interventions pendant cette séquence d'une heure de dragage difficile (voir Tableau 2). Les actions sont courtes (quelques secondes), peu variées principalement les deux manettes du treuil de cardan et de bec - et très répétitives, jusqu'à 135 fois le treuil du bec. L'opération en mode manuel entraîne ici une majoration des corrections avec les manettes, ce qui amène à une configuration identique à celle de la drague stationnaire (qui ne dispose pas du mode automatique). L'assistant de pont de la drague aspiratrice en marche est ici plus fréquemment sollicité que son homologue - plus de trois actions par minute (contre deux par minute sur la stationnaire). 


\begin{tabular}{lc}
\hline Description de l'activité & Nombre d'actions \\
\hline Treuil du cardan (manette) & 45 \\
Treuil d'élinde (manette) & 3 \\
Treuil du bec (manette) & 135 \\
Pompe (mise en marche/arrêt par bouton poussoir) & 7 \\
Communication orale & 3 \\
Acquitter les alarmes (bouton poussoir) & 3 \\
\hline Total & 196 \\
\hline
\end{tabular}

Tableau 2 : La charge de travail de l'assistant de pont sur la drague aspiratrice en marche sur une heure d'activité (Observation du 30/10/05, dragage du port de Boulogne sur Mer).

Tableau 2: Deck assistant's work load on the pump dredger motion ship during one hour's (Observations on the 30/10/05, dredging in Boulogne sur Mer harbour).

Au final, l'analyse de l'activité des assistants de pont fait ressortir des interventions courtes et nombreuses au cours de situations de dragage qui s'étendent sur de longues durées. Que cela soit sur la drague aspiratrice en marche ou la drague stationnaire, les multiples actions de l'assistant de pont requièrent le maintien d'un niveau de vigilance élevé, au risque d'obstruer le système d'aspiration, d'endommager le matériel et de nuire aux membres d'équipage. La tâche du dragueur est sensiblement complexifiée sur des fonds marins rocheux ou jonchés de débris. Dans ce cas, il va être fortement sollicité sur le plan cognitif et pourrait ressentir des effets de stress ponctuel et/ou de fatigue.

\section{5.- Discussion}

$\mathrm{Au}$ cours de cette étude exploratoire, les analyses de nos indicateurs psychologique et physiologique sont apparues plus concordantes que divergentes, concluant conjointement à un niveau de complexité de l'activité en lien avec la nature du fond sous-marin. Néanmoins, les différents niveaux d'analyse adoptés tendraient à être complémentaires dans la caractérisation de ces situations de dragage difficiles :

- l'observation ethnographique a permis d'inventorier précisément les nombreuses actions brèves de l'assistant de pont au sein de son contexte général d'intervention ;

— les entretiens compréhensifs ont appréhendé la notion de vigilance et les multiples focalisations attentionnelles (Lachaux, 2011) du dragueur induisant fatigue et lassitude ;

— la variabilité de la fréquence cardiaque a mis en relief l'intensité du dragage et l'éventuelle survenue de stress ponctuel par sollicitation de la branche orthosympathique du système nerveux avec un marquage précis des instants de survenue de ces événements.

Cette approche «intégrée » pourrait fournir de nouvelles perspectives méthodologiques dans l'étude d'objets de recherche ergonomiques complexes, où les dimensions psychologique et physiologique s'avèrent fortement intriquées, tout comme dans le cas de la fatigue.

La fatigue demeure un «concept-problème » sur le terrain, notamment en milieu maritime, puisqu'elle figure en tant que cause récurrente dans l'accidentologie (BEA- mer, 2007). La fatigue englobe diverses notions : les rythmes biologiques (sommeil et vigilance), le stress, ou encore la charge de travail. Celles-ci ont été abordées au cours de cette étude sur les dragues. Ces catégories n'en restent pas moins hétérogènes et dotées de contours assez flous, puisque recoupant différents champs scientifiques, de la physiologie à la psychologie. À titre d'exemple, il est possible d'appréhender l'état de vigilance des gens de mer aussi bien à partir d'électro-encéphalogrammes (La Giclais, 2009), qu'en se basant sur les perceptions subjectives d'un individu (Gander, Van Den Berg, \& Signal, 2008 ; Tirilly \& Foret, 2000).

Pareillement, la charge de travail a un coût aussi bien physiologique que psychologique 
(Theureau \& Jourdan (2002). Les tâches diverses sont à exécuter simultanément, ou dans un laps de temps très rapproché. Le dragueur est alors obligé de sélectionner les informations pertinentes, et de reléguer au second plan des données jugées moins essentielles. Au niveau neurophysiologique, cette focalisation attentionnelle sur un domaine précis se réalise aux dépens d'autres sources d'information (Lachaux, 2011 ; Siegel, 2002).

Cet afflux d'informations simultanées émanant de l'environnement n'est pas nécessairement contraignant, même si ces circonstances pourraient parfois générer une «surcharge mentale » et provoquer des incidents (Theureau \& Jourdan, 2002). Néanmoins, les marins qui évoluent quotidiennement en autonomie sur leur embarcation ne semblent pas privilégier la prise de risque. Il s'agit plutôt de programmer les différentes actions en fonction de la pression temporelle, par une hiérarchisation entre les « attracteurs cognitifs » (ibid.).

Dans le cadre de cette étude, nous avons également identifié la présence de plusieurs situations stressantes, en recoupant les enregistrements de variabilité de la fréquence cardiaque et nos observations. Malgré tout, nous n'avons relevé que très peu d'incidents. Pour Morel, Amalberti et Chauvin (2008), les gens de mer vont savoir s'adapter aux conditions et à leur charge de travail afin de maintenir un niveau de sécurité maximal. Ce comportement expert s'est développé à travers leur expérience des situations à risque et des tâches stressantes à gérer quotidiennement. Cette « routinisation » offre une stabilité accrue à leurs états émotionnels (Le Scanff, 2000). Ces analyses corroborent les conclusions de l'étude longitudinale de Gunnarsson, Bäck, Jones et Olsson (2004). Ces derniers ont suivi un équipage engagé sur un tour du monde durant 9 mois. Ils montrent une diminution des marqueurs biologiques du stress au fur et à mesure de la compétition. Cependant, méthodologiquement, ces auteurs se sont restreints dans leur approche du stress aux dosages hormonaux ou à l'analyse des perceptions subjectives de l'individu.

Il serait possible d'éclairer l'hétérogénéité entre vigilance, charge de travail et stress par l'observation de décalages, entre la fatigue physiologique de l'organisme d'une part, et la perception qu'en aurait un individu d'autre part. Même si nous n'avons pu repérer de tels décalages dans notre étude exploratoire sur les dragues, l'articulation de la physiologie et de la psychologie réclame un espace commun, sur lequel devrait se construire l'observatoire. C'est un espace commun de ce type que nous avons ici tenté de mettre en place, à travers des interfaces méthodologiques opératoires.

Cette méthodologie «intégrée» pourrait être employée avec profit pour traiter des problèmes de fatigue, et donc de sécurité, à bord des navires. D'autant plus que l'état de fatigue général de l'organisme peut être également appréhendé à travers la variabilité de la fréquence cardiaque (Jouanin, Dussault, Pérès, Satabin, Piécard \& Guézennec, 2004 ; Pichot, Bourin, Roche, Garet, Gaspoz, Duverney et al., 2002).

\section{6.- Conclusion}

Cette étude exploratoire a poursuivi un objectif ergonomique, tentant d'analyser l'activité humaine dans un milieu difficilement accessible (fortes contraintes de l'environnement). Nous avons tenté de collecter des données provenant de sources variées, puis de les faire dialoguer entre elles de façon scientifiquement opératoire. Cet aménagement méthodologique offre l'avantage de confronter des points de vue physiologique et psychologique, dans le but de produire une analyse de l'activité "écologiquement pertinente ».

En termes de conception ergonomique, même si nos observations n'ont pas mis à jour de situations problématiques majeures, il nous paraît souhaitable d'améliorer la sécurité à bord de ces navires. Cette remédiation passerait avant tout par une meilleure répartition de la charge de travail associée au poste de dragueur. En effet, nos résultats rendent compte d'une forte fluctuation de la difficulté du dragage selon la nature du fond à draguer, que ce 
soit à travers les observations, les entretiens, ou les interprétations des enregistrements de la fréquence cardiaque. Ces informations sur les fonds sous-marins étant disponibles de manière préalable au dragage, il serait judicieux d'alterner, dans la mesure du possible, les zones connues comme difficiles avec des zones plus aisées. Cette alternance aurait pour but de répartir les efforts des marins au cours de la tâche (vigilance et stress), et de tendre à l'instauration d'une constance dans la difficulté du travail.

Nous projetons dorénavant de travailler sur les processus de fatigue en milieu maritime avec une «méthodologie croisée » de ce type, en adjoignant aux enregistrements simultanés à l'activité, des mesures de la variabilité de la fréquence cardiaque sous forme d'enregistrements standardisés (Sennegon, Grison, \& Buttelli, 2009a; 2009b). Une confrontation de ces mesures physiologiques aux perceptions subjectives (observation ethnographique et entretiens) s'avérerait peut-être ainsi d'une fécondité accrue.

\section{BIBLIOGRAPHIE}

Bachelor, A., \& Joshi, P. (1986). La méthode phénoménologique de recherche en psychologie. Québec: Presses de l'Université Laval.

Becker, H.S. (1958). Problems of Inference and Proof in Participant Observation. American Sociological Review, 23(6), 652-660. Reproduit in D. Céfaï, L'enquête de terrain (pp. 350-362). Paris: La Découverte.

Béguin, P. ,\& Clot, Y. (2004). L'action située dans le développement de l'activité. @ ctivités, 1(2), 2749.

Borresen, J., \& Lambert, M.I. (2007). Changes in heart rate recovery in response to acute changes in training load. European Journal of Physiology, 101, 503-511.

Bureau Enquêtes Accidents mer (2007). 1997 - 2007, Dix ans d'expérience, d'enquêtes, d'études et de recommandations de sécurité. [On-line]. Available: http://www.beamer-France.org/

Cassirame, J., Tordi, N., Mourot, L., Rakobowchuk, M., \& Regnard, J. (2007). L'utilisation d'un nouveau système d'enregistrement de fréquence cardiaque battement à battement pour l'analyse traditionnelle de variabilité de fréquence cardiaque. Science \& Sports, 22, 238-242.

Chassagnieux, A., Garrigou, A., Lortie, M., Teiger, C., Carballeda, G., Pargade, V., Pasquereau, P., \& Perraut, C. (2012). Evaluations des facteurs de pénibilité du travail et évolutions technicoorganisationnelles : un défi pour l'ergonomie - le cas des imprimeurs-rotativistes de la presse quotidienne à trente ans de distance. Pistes, 14(1).

Direction générale de la mer et des transports, (2007). Rapports et statistiques 2007 : Les accidents du travail maritime. [On-line]. Available: http://www.mer.gouv.fr/

Duval, M. (1998). Ni morts, ni vivants : marins! - Pour une ethnologie du huis clos. Paris: Presses Universitaires de France.

Gander, P., Van Den Berg, M., \& Signal, L. (2008). Sleep and sleepiness of fishermen on rotating schedules. Chronobiology International, 25(2), 389-398.

Geslin, P. (2002). Les formes sociales d'appropriations des objets techniques, ou le paradigme anthropotechnique. Ethnographiques.org, 1 [On-line]. Available: http://www.ethnographiques.org/

Gold, R.I. (1958). Roles in Sociological Field observations. Social Forces, 363, 217-233. Reproduit in D. Céfaï (Ed.), L'enquête de terrain (pp. 340-349). Paris: La Découverte.

Grison, B. (2004). Des Sciences Sociales à l'Anthropologie Cognitive. Les généalogies de la Cognition Située. @ ctivités, 1(2), 26-34.

Grossmann, A., \& Morlet, J. (1984). Decomposition of hardy function into square integrable wavelets of constant shape. SIAM J Math, 15, 229-242.

Gunnarsson, L.G., Bäck, H., Jones, I., \& Olsson, T. (2004). Stress recovery during an ocean boat race. Stress and health, 20, 165-171.

Guzzeti, S., Signorini, M., Cogliati, C., Mezzeti, S., Porta, A., Cerutti, S., \& Malliani, A. (1996). Nonlinear dynamics and chaotic indices in heart rate variability of normal subjects and hearttransplanted patients. Cardiovascular Research, 31, 441-446.

Hazelhurst, B. (1999). Fishing for cognition: an ethnography of fishing practice in a community on 
the west coast of Sweden. Thèse de Doctorat non publiée. University of California, San diego.

Hjortskov, N., Rissén, D., Blangsted, A.K., Fallentin, N., Lundberg, U., \& Sogaard, K. (2004). The effect of mental stress on heart rate variability and blood pressure during computer work. European Journal of Applied Physiology, 92, 84-89.

Jeffroy, F., Theureau, J., \& Haradji, I. (2006). Relation entre activité individuelle et activité collective. Toulouse: Octarès.

Jouanin, J.C., Dussault, C., Pérès, M., Satabin, P., Piécard, C., \& Guézennec, Y. (2004). Analysis of heart rate variability after a ranger training course. Military Medicine, 169, 583-587.

Journé, B. (2006). Observer la sûreté et en rendre compte : éléments de méthode. In F. Jeffroy, J. Theureau, \& Y. Haradji (Eds.). Relation entre activité individuelle et activité collective (pp. 95118). Toulouse: Octarès.

La Giclais, B. de (2009). Le sommeil particulier des navigateurs solitaires à la voile en course transocéanique pour une bonne vigilance. In Quatorzièmes journées de la médecine des gens de mer. Paris. [On-line]. Available: http://www.mer.gouv.fr/IMG/doc/de_la_GICLAIS_cle098f9b.doc

Lachaux, J.P. (2011). Le cerveau attentif. Paris: Odile Jacob.

Laperrière, A. (1992). L'observation directe. In B. Gauthier (Ed.), Recherche Sociale : de la problématique à la collecte des données (pp. 251-272). Sillery: Presses de 1'Université du Québec.

Leplat, J. (2000). L'analyse psychologique de l'activité en ergonomie. Toulouse: Octarès.

Leplat, J. (2002). De l'étude de cas à l'analyse de l'activité. Pistes, 4(2), 1-31.

Leplat, J. (2008). Repères pour l'analyse de l'activité en ergonomie. Paris: Presses Universitaires de France.

Le Roy, Y., \& Breuille, P. (1997). La fiabilité humaine dans la sécurité en mer. In Troisièmes journées de la médecine des gens de mer. Paris.

Le Scanff, C. (2000). Les aventuriers de l'extrême. Paris: Calmann-Lévy.

Maline, J., \& Dorval, P. (1991a). Analyse du travail d'un équipage : prise en compte de l'activité des marins pêcheurs. Le Travail Humain, 54(2), 165-184.

Maline, J., \& Dorval, P. (1991b). Analyse de travail du marin pêcheur et fréquence cardiaque. Archives des maladies professionnelles de médecine du travail et de sécurité sociale, 52(1), 7-15.

Martinmäki, K., Häkkinen, K., Mikkola, J., \& Rusko, H. (2008). Effects of low-dose endurance training on heart rate variability at rest and during an incremental exercise test. European Journal of Physiology, 104, 541-548.

Minguy, J., \& Chauvin, C. (1998). Recherche en ergonomie des activités maritimes. In Actes du colloque Recherche et Ergonomie. Toulouse.

Monod, H., \& Kapitaniak, B. (2003). Ergonomie. Paris: Masson.

Morel, G., Amalberti, R., \& Chauvin, C. (2008). Safety and Resilience: Articulation and Difference The example of the professionnal fishing industry. Human Factors, 50(1), 1-16.

Perini, R., \& Veicstenas, A. (2003). Heart rate variability and autonomic activity at rest and during exercise in various physiological conditions. European Journal of Applied Physiology, 90, 317325.

Pichot, P., Bourin, E., Roche, F., Garet, M., Gaspoz, J.M., Duverney, D., Antoniadis, A., Lacour, J.R., \& Barthélémy, J.C. (2002). Quantification of cumulated physical fatigue at the workspace. European Journal of Physiology, 445, 267-272.

Pierpont, L.G., Stolpman, D.R., \& Gornick, C.C. (2000). Heart rate recovery post-exercice as an index of parasympathetic activity. Journal of the autonomic nervous system, 80, 169-174.

Rajendra Acharya, U., Paul Joseph, K., Kannathal, N., Choo Min Lim, \& Jasjit Suri, S. (2006). Heart rate variability : a review. Medical and Biological Engineering, 44(12), 1031-1051.

Salembier, P., Theureau, J., Zouinar, M., \& Vermersch, P. (2001). Action/Cognition située et assistance à la coopération. In Douzièmes journées francophones d'ingénierie des connaissances. Grenoble.

Samar, V., Bopardikar, A., Rao, R., \& Swartz, K. (1999). Wavelet analysis of neuroelectric waveforms: A conceptual tutorial. Brain and Language, 66, 7-60. 
Schütz, A. (2009). Symbole, réalité et société (1955). In A. Schütz, \& C. Schrecker (Eds.), Contribution à la sociologie de l'action (pp. 51-133). Paris: Hermann.

Sennegon, B., Grison, B., \& Buttelli, O. (2009a). Les Processus de fatigue en milieu maritime, approche psychophysiologique. In Quatorzièmes journées de la médecine des gens de mer. Paris. [On-line]. Available: http://www .mer.gouv.fr/

Sennegon, B., Grison, B., \& Buttelli, O. (2009b). Les Processus de fatigue des skippers en solitaire, approche psychophysiologique. In XIIIème Congrès International ACAPS. Lyon.

Sève, C., Saury, J., Theureau, J., \& Durand, M. (2002). La construction de connaissances chez des sportifs de haut niveau lors d'une interaction compétitive. Le Travail Humain, 65(2), 159-190.

Siegel, J. (2002). The neural control of sleep and waking. New York, Wien: Springer Verlag.

Strauss, A., \& Corbin, J. (1990). Grounded theory Research: Procedures, Canons, and Evaluative Criteria. Qualitative Sociology, 13(1), 3-22. Reproduit in D. Céfaï (Ed.) L'enquête de terrain (pp. 363-379). Paris: La Découverte.

Task Force of European Society of Cardiology and the north American society of pacing and electrophysiology, (1996). Heart rate variability: standards of measurement, physiological interpretation, and clinic use. European Heart Journal, 17, 354-381.

Theureau, J. (2004). L'hypothèse de la cognition (ou action) située et la tradition d'analyse du travail de l'ergonomie de langue française. @ ctivités, 1(2), 11-25.

Theureau, J. (2006). Le cours d'action : méthode développée. Toulouse: Octarès.

Theureau, J., \& Jeffroy, F. (1994). Ergonomie des situations informatisées. Toulouse: Octarès.

Theureau, J., \& Jourdan, M. (2002). Charge mentale : notion floue et vrai problème. Toulouse: Octarès.

Tirilly, G., \& Foret, J. (2000). Évolution du rythme activité/repos et de la vigilance subjective d'un équipage de marins pêcheurs. In Congrès SELF 2000, pp. 550-560.

Varela, F. (1996). Invitation aux Sciences Cognitives. Paris: Point Seuil.

Vermersch, P. (2000). Conscience directe et conscience réfléchie. Intellectica, 2(31), 269-311.

Vermersch, P. (2006). Contrepoint. L'explicitation phénoménologique à partir du point de vue en première personne. In F. Jeffroy, J. Theureau, \& I. Haradji (Eds.), Relation entre activité individuelle et activité collective (pp. 129-146). Toulouse: Octarès.

\section{RESUME}

Cette étude a pour objectif d'explorer une méthodologie recoupant deux ordres de données, psychologiques et physiologiques, dans le cadre d'une analyse ergonomique de l'activité. Deux terrains d'étude, situations «naturelles », ont été investis: une drague aspiratrice stationnaire et une drague aspiratrice en marche, où nous nous sommes focalisés sur l'assistant de pont en charge de l'activité de dragage.

Au niveau psychologique, notre méthode a consisté à croiser des observations ethnographiques avec des entretiens individuels compréhensifs. Au niveau physiologique, des mesures de la fréquence cardiaque (intervalle R-R) ont été réalisées à travers des enregistrements simultanés à l'activité.

Nos résultats découlent alors de la confrontation de ces sources de données « objectives » et «subjectives ». Au poste du dragueur, la charge de travail paraît conséquente avec des actions courtes et nombreuses. Il semble nécessaire de maintenir un niveau de vigilance élevé, en fonction des différents paramètres à prendre en considération et des régulations multiples à fournir lors de la tâche. L'activité se complexifie selon la nature des fonds sous-marins, avec la survenue de nombreuses variations et l'apparition possible de stress lors de dragages sur fond chaotique. 
Cette prise en compte «intégrée » de l'activité humaine devrait permettre en retour l'amélioration de la sécurité des marins.

\section{MOTS CLES}

analyse de l'activité, psychophysiologie, variabilité de la fréquence cardiaque, étude de cas, sécurité

\section{REFERENCEMENT}

Sennegon, B., Grison, B., Ravier, Ph., \& Buttelli, O. Analyse de l'activité à bord de dragues aspiratrices: une méthodologie exploratoire combinant données psychologiques et physiologiques. Activités, 9(2), 112-132, http://www.activites.org/v9n2/v9n2.pdf

Article soumis en août 2011, accepté pour publication le 2 juillet 2012 